\title{
Development of a Smart System for COVID-19 Guideline Compliance Verification
}

\author{
Rashmi Welekar', Manjiri Vairagade², Mohit Sawal ${ }^{3}$, \\ Shreya Rathi ${ }^{4}$, Shrijeet Shivdekar ${ }^{5}$, Siddhi Belgamwar ${ }^{6}$ \\ Department of Computer Science \& Engineering, Shri Ramdeobaba College of Engineering \\ and Management, Katol Road, Gittikhadan, Nagpur, 440013, Maharashtra, India
}

\section{ABSTRACT}

The important reasons for the rapid spread of the novel coronavirus (2019-nCoV) was the lack of awareness of infected individuals about their health due to the 14 day incubation period of the virus and the subsequent un-intentional transmission, large scale ignorance of social distancing guidelines, and improper sanitary precautions and health. In this paper, we describe the development process of a system based solution for individuals to do Covid-19 susceptibility test using the Sp02 oxygen level detection test, to detect symptoms with higher levels of accuracy. We also discuss various other implementation features to prevent the unaware spread of the virus including providing details to the user regarding government guidelines related to containing transmission of the virus, alerts to the user about periodic maintenance of sanitary guidelines, social distancing, and notifying the user on non-compliance of above features. Finally, we discuss possible future extensions to stricter the measures taken up by the user to prevent any mistakes- like a front-camera based mask authentication approach, and alerting the user on entering crowded areas based on Bluetooth crowd sensing. Also, this system will be further developed into a mobile application.

KEY WORDS: COVID-19, COMPLIANCE, SP02 OXYGEN LEVEL MEASUREMENT, GOVERNMENT GUIDELINES, ALERTS FOR SANITARY GUIDELINES, SOCIAL DISTANCING, AND FACE MASK.

\section{INTRODUCTION}

The severe acute respiratory syndrome coronavirus-2 (SARS-CoV-2) has triggered a worldwide phenomenon which led to large scale repercussions in health and economy. The WHO declared the coronavirus as a pandemic on 11th march, especially that of the category known as Public Health Emergency of International Concern (PHEIC).[Mahalmani VM,2020] As of 14th August 2020, the number of cases recorded in India were

\section{ARTICLE INFORMATION}

*Corresponding Author: welekarr@rknec.edu

Received 15th Oct 2020 Accepted after revision 25th Dec 2020

Print ISSN: 0974-6455 Online ISSN: 2321-4007 CODEN: BBRCBA

Thomson Reuters ISI Web of Science Clarivate Analytics USA and Crossref Indexed Journal

\section{Clarivate
Analytics}

NAAS Journal Score 2020 (4.31)

A Society of Science and Nature Publication,

Bhopal India 2020. All rights reserved.

Online Contents Available at: http//www.bbrc.in/

Doi: $h t t p: / / d x$.doi.org/10.21786/bbrc/13.14/46 approximately 2.46 million and the number of deaths were 48,040. The widespread influence of the coronavirus pandemic has resulted in proactive steps taken by the Government to contain the spread in order to prevent further swathes of the population from getting infected, because of the case fatality rate to be $2 \%$ as of April 2020[Fauci ,2020], and 3.02\% as of September 2020. One of the most important steps taken by the government to control the transmission is comprehensive testing. Testing vulnerable hordes of the population through various measures like RT-PCR, serologic testing, lateral flow assay, etc. have proven to effectively identify Covid-19 positive and negative audiences. The further clamping down on spread is ensured through contact tracing.

However, the crucial factor which has led to an increase in the number of cases is the incubation period of the virus, which is 14 days[Jantien A Backer,2020]. This incubation period signifies the approximate time required 
for the emergence of symptoms in a COVID-19 positive individual. In these 14 days, ignorance and not paying attention to symptoms may lead to the persons in contact with the COVID-19 positive individual being susceptible to infection, which is known as the asymptomatic transmission of the virus.

[Bai Y,2020][ Gao M,2020] Special care has to be taken to monitor the health conditions and oxygen levels of patients with hypertension and diabetes mellitus because of their increased susceptibility to the disease [Fang L,2020]. The RT-PCR testing method, despite its high accuracy, leans towards the non-feasible side for economically poor households. We can leverage the wide smartphone base and internet connectivity in India, even in poorer households, to come up with a solution to determine the susceptibility of an individual towards COVID-19 through oxygen level detection in the blood. [ Xie,2020] Thus, in times of COVID-19, oxygen level monitoring has become especially important, because of low oxygen levels being an important indicator of the severe cases of COVID-19, with the individuals not being immediately aware of it.

A lot of asymptomatic patients also suffer from extraordinarily low blood-oxygen levels, or hypoxia without even realizing, which has made the prognosis of the infected individuals difficult. This is because low oxygen levels do not necessarily point towards any visible respiratory problems. This silent depletion of oxygen levels in the body of asymptomatic patients may ultimately lead to cardiac arrest. Since most asymptomatic patients don't prohibit any obvious symptoms of low concentration of oxygen levels, it becomes all the more essential to closely monitor the oxygen saturation, in the early stages of the disease. Patients are unaware of the depletion of oxygen as they are able to breathe comfortably[Tobin,2020].

However, they may experience confusion, impaired psychomotor performance, and even euphoria, none of which can be clearly linked to oxygen deprivation in the body. The slow drop in oxygen levels has shed light on the importance of a device to measure the percentage of oxygen in the blood and the heart rate. Normally, a person's oxygen saturation ( $\mathrm{SpO2}$ ) is around 94-96 percent and becomes a cause for concern when it starts dropping below 92 percent. Apart from this, violation of certain preventive measures can lead to the transmission of the virus. We aim to develop a system that will help in keeping track of a user's health status and provide him with the ability to test himself against the virus along with other features to verify his compliance against the measures set. The system can then, in later stages, be developed into a mobile application for the ease of use and availability to every smartphone owner.

Literature Review: The overall understanding of the COVID-19 pandemic and its repercussions, fatality rate, incubation period and the mode of asymptotic transmission was comprehensively explained by Mahalmani et al(2020) in [Mahalmani VM,2020],
Fauci, Anthony, Lane, and Redfield(2020) in [Fauci, 2020], Backer, Klinkenberg and Wallinga(2020) in [Jantien A Backer,2020] and Bai, Yao, Wei et al.(2020) in [Bai Y,2020] respectively. To get an idea about the feasibility and existing systems, the paper by Jhunjhunwala(2020) in [Jhunjhunwala A,2020] provided sufficient information about the Arogya Setu app and the possible ways to leverage the vast smartphone user base. Fang, Karakiulakis, and Roth(2020) in [Fang L,2020] conveyed the urgency to develop a system that could determine the susceptibility of high risk patients (diabetes and hypertension), considering the high case fatality rate. The Sp02 level detection module was motivated by the importance of monitoring Sp02 levels in COVID-19 patients as suggested by Xie, Jiang, et al.(2020) in p8[ and the fact that levels above $90 \%$ statistically pointed to a reduced mortality rate.

Another motivation to develop the module was the baffling phenomenon of "happy hypoxia", by virtue of which a high risk patient may seemingly be unaware of his low oxygen concentration and subsequently, low Sp02 levels, and may be at risk according to Tobin, Martin, Franco et al.(2020) in [Tobin,2020]. To develop this module of determining blood oxygen saturation using smartphones, Lamonaca, Carni, Grimaldi et al.(2015) in [F. Lamonaca,2015] provided useful insights on processing of change of light intensity in the video frames at the fingertips of the patient.Kanva, A.K., Sharma, C.J., \& Deb, S. (2014) in [Kanva,2014], narrowed down on the extraction of data of recorded variations in colour signals on a fingertip placed in contact with the optical sensor and Bui, Nguyen, Nguyen et al.(2020) in [Nam Bui,2020] gave systematic insights about measuring Sp02 by exploiting wavelength separation and chromophore compensation. The motivation and feasibility for development of the bluetooth proximity sensing module and its importance to return to normalcy in times of pandemic was conveyed by Xia, Ye and Lee(2020) in [Xia,2020]. Finally, the understanding of the phenomenon of mass immunization and how use of AI, digital monitoring and repurposing of existing other resources with IoT, NFC and GPS protocols will optimize and handle the major challenges was succinctly conveyed by Indani et al.(2020) in [Ashish Indani,2020]

Existing System: The Arogya Setu app, leverages the large smartphone base and the expansive coverage of telecom network services and internet facilities, to augment the efforts taken by administrators and coordinators to better manage the pandemic at a local level[Jhunjhunwala A,2020]. The individuals who are suspected COVID 19 positive based on the assessment quiz are asked about location geographically through GPS and bluetooth, and users of the app around this suspected individual are given alerts and notification, citing their risk category on basis of proximity to that individual. The App provides various other features as well, by virtue of its augmentation with government initiatives to reduce the workload and risks of physical presence, like issuing e-passes. The convenient user interface makes the app very handy to use. The app does 
not provide any sort of symptom detection test, which could benefit the large user base of this app. Also, the app does not periodically remind the user about maintaining sanitary precautions or social distancing guidelines or giving the user an idea about the individuals who come in contact with him during any outdoor activity, thus rendering him less aware of the degree of safety of any place visited by the user.

Proposed System: Our system primarily relies on $\mathrm{SpO} 2$ based oxygen saturation detection test to classify the susceptibility of users as risk-averse, or risk-negative. The result obtained would be supported by predicting the susceptibility depending upon the symptoms observed in the user. Periodic alerts will be given to remind the user to comply with the preventive measures. In case of a user stepping out of his home, he would be prompted to take a photo of himself so as to check whether the user is wearing a mask or not. If not, he will be asked again and again until the mask is detected by the system. When in crowded areas, social distancing will be monitored with the help of bluetooth by checking the nearest devices in an area of some radius. Government guidelines will be displayed to the user so as to comply with them. If the user is found violating the preventive measures, he will be notified immediately. A dashboard will be maintained for the user to check his health parameters and other details.

Since the patients may gradually become used to the slow concentration of oxygen level in the body, a pulse oximeter or a device which measures the oxygen concentration levels can come handy in spotting when the oxygen concentration level starts dropping. This may help in providing the patient with timely oxygen supply before things become critical and even help in early detection of COVID pneumonia when there are minor symptoms.

\section{METHODOLOGY}

To develop the above mentioned system and incorporate different features, we make use of the SpO2 concept that measures the oxygen saturation level in the blood. This will help in detecting the presence of virus in the human body. Monitoring social distancing in crowded areas with the help of bluetooth, detection of face masks before stepping out of the house, dashboard to keep track of health parameters are some of the other features that will be supported by the system.

\section{- Sp02 Level Measurment}

We primarily rely on the pulse oximetry measurement using smartphones to determine the SpO2 levels present in the blood.[ F. Lamonaca,2015]The oximeter produces two wavelengths of light which pass through the finger/ palm and the unabsorbed light is measured by a photo detector. Pulse oximetry relies on the principle that the blood absorbance of red light changes with variance in degree of oxygenation. The receiver end esignal is divided into DC and AC components. The DC component is due to tissue background, venous blood and constant part of arterial blood flow. The DC component is subtracted while the AC component is amplified. The DC and AC parts can be used to calculate the SpO2 level in the blood, according to the formula given in Fig. 1.

$$
S_{p} O_{2}=A-B \frac{A C_{R E D} / D C_{R E D}}{A C_{B L U E} / D C_{B L U E}}
$$

\section{Figure 1: Sp02 based oxygen saturation detection test}

Where, A and B are parameters that can be obtained by matching the Sp02 graph obtained by a standard pulse oximeter.[ Ding,2018] The user has to keep his finger/ palm on his smartphone's camera as shown in Fig. 2. This is the first step of the algorithm involving the recording of a small video clip of around 20 seconds. Around 20 seconds is essential for the calculated values to stabilize. For this particular implementation of the algorithm to work, the white light will fall on the finger/palm and then the image of the finger/palm will be received by the phone's camera lens. After placing a finger/palm on the lens, the user has to make sure that the image produced on the screen is sufficiently bright. He will then press the Start button to start recording the clip. Second Step: When around 20 seconds will pass, the recording will automatically stop. Once this happens, a new screen will be launched in the app. The first 600 frames from the clip (corresponding to around 20 seconds of video) are extracted.The retracted frames shall look like the one shown in Fig. 3

Figure 2: Position of the finger while

Figure 3: Frame extracted from video

Figure 4: Sp02 capturing video Methodology

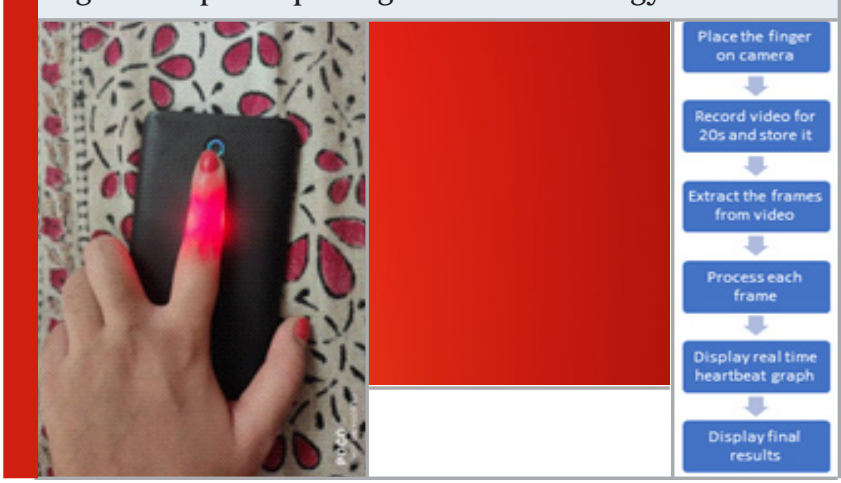

Third Step: After the extraction of the frames, they will be parsed into an image holder where they will be processed one by one. Each frame will be resized for reduced computation time. Each frame will be separated into its red and blue constituents. The following parameters will be calculated for each image: mean of red color component (mr), mean of blue color component (mb), standard deviation of red color component (sdr) and the standard deviation of blue color component (sdb). Here, $\mathrm{mr}$ corresponds to the red DC value, $\mathrm{mb}$ to the blue DC value, sdr to the red $\mathrm{AC}$ value and sdb to the blue $\mathrm{AC}$ value. These values will be used to calculate the $\mathrm{SpO} 2$ 
level. The calculated average Sp02 value of all the 600 frames will be then displayed and an average value obtained from Sp02 graph will be saved for further use.

Final Step: When all the required parameters will be obtained, the following suggested actions will be shown to the user:

1. Sp02 calculated between 96 and 99 Result: Healthy 2. SpO2 calculated $<92$ Result: The user should try again, if values persist, he should cross-check his values with standard equipment.

If the value also persists on standard equipment, then the user should seek medical attention, because these levels indicate low levels of oxygen concentration in the blood. [ Nam Bui,2020] This methodology is diagrammatically explained in Fig. 4.

To support the above mentioned results, a machine learning model will be trained to apprise the user of his health status. Inputs such as fever, cough, sore throat, tiredness, etc would be taken from the user and applied against the model. Depending upon the output obtained, the final result will be shown to the user as whether he is safe or if he needs to seek medical aid.

\section{- Mask Detection}

1. Access location of the user.

2. If the location is other than the home location, prompt the user to capture his image.

3. This image will undergo a series of techniques of image processing.

4. Based on the processed image, the app will output whether the user has worn any mask or not.

5. If the user has not worn a mask, then an alert will be given to the user to wear the mask and retake his photo until the mask is detected to ensure his safety and will be good to go after wearing the mask.

\section{- Social Distancing Using Bluetooth}

1. The user grants the necessary permissions to the app like bluetooth, location, etc

2. We create a BluetoothServerSocket to listen for connection requests from other devices.

3. Compute the number of contacts every 30 seconds. This compensates for things like differences in bluetooth advertising rate.

4. Serialize these contacts into a Gson file with the appropriate name, signal strength and device address for each contact.

5. We can convert this Gson file to Json and send it to our server to store user information

6. count the number of space-separated addresses in the contact list and display in today's score.[ Xia,2020]

\section{CONCLUSION}

We aim to develop a system that will have features like detection of virus in the human body using Sp02 and other visible symptoms like fever,cough,sore throat,tiredness,etc., verification of user compliance against preventive measures like social distancing when moving through public places or crowded areas and use of face masks while stepping out of the house. This system will be further developed into a mobile application for the ease of use and availability to every smartphone owner. This system shall help mankind technologically while such dire situations demanding medical aid prevails.

\section{REFERENCES}

Ashish Indani, Dr. Kedar Mehta, Devraj Goulikar, Pratibha Potare, Vikrant Singh, Gaurav Srivastava \&t Aejaz Hussain.Preparing For Intelligent Infrastructure For Mass Immunization Related Supply Chain And Monitoring Process. Journal of Hospital Pharmacy, Supp-(15-3) A-3(2020)

Bai Y, Yao L, Wei T, et al. Presumed Asymptomatic Carrier Transmission of COVID-19. JAMA. 2020;323(14):14061407. doi:10.1001/jama.2020.2565

Ding, Xinyi, Damoun Nassehi, and Eric C. Larson. "Measuring Oxygen Saturation With Smartphone Cameras Using Convolutional Neural Networks." IEEE journal of biomedical and health informatics 23.6 (2018): 2603-2610.

F. Lamonaca, D. L. Carnì, D. Grimaldi, A. Nastro, M. Riccio and V. Spagnolo, "Blood oxygen saturation measurement by smartphone camera," 2015 IEEE International Symposium on Medical Measurements and Applications (MeMeA) Proceedings, Turin, 2015, pp. 359-364, doi: 10.1109/MeMeA.2015.7145228.

Fang L, Karakiulakis G, Roth M. Are patients with hypertension and diabetes mellitus at increased risk for COVID-19 infection? [published correction appears in Lancet Respir Med. 2020 Jun;8(6):e54]. Lancet Respir Med. 2020;8(4):e21. doi:10.1016/S22132600(20)30116-8

Fauci, Anthony S., H. Clifford Lane, and Robert R. Redfield. "Covid-19-navigating the uncharted." (2020): 1268-1269.

Gao M, Yang L, Chen X, et al. A study on infectivity of asymptomatic SARS-CoV-2 carriers. Respir Med. 2020;169:106026. doi:10.1016/j.rmed.2020.106026.

Jantien A Backer, 1 Don Klinkenberg, 1 and Jacco Wallinga 1 , 2 Incubation period of 2019 novel coronavirus (2019-nCoV) infections among travellers from Wuhan, China, 20-28 January 2020

Jhunjhunwala A. Role of Telecom Network to Manage COVID-19 in India: Aarogya Setu [published online ahead of print, 2020 Jun 2]. Transactions of the Indian National Academy of Engineering. 2020;1-5. doi:10.1007/s41403-020-00109-7

Kanva, A.K., Sharma, C.J., \&t Deb, S. (2014). Determination of SpO2 and heart-rate using smartphone cameras. 
Proceedings of The 2014 International Conference on Control, Instrumentation, Energy and Communication (CIEC), 237-241.

Mahalmani VM, Mahendru D, Semwal A, Kaur S, Kaur H, Sarma P, Prakash A, Medhi B. COVID-19 pandemic: A review based on current evidence. Indian J Pharmacol 2020;52:117-29

Nam Bui, Anh Nguyen, Phuc Nguyen, Hoang Truong, Ashwin Ashok, Thang Dinh, Robin Deterding, and Tam Vu. 2020. Smartphone-Based Sp02 Measurement by Exploiting Wavelengths Separation and Chromophore Compensation. ACM Trans. Sen. Netw. 16, 1, Article 9
(February 2020), 30 pages.

Tobin, Martin J., Franco Laghi, and Amal Jubran. ”Why COVID-19 silent hypoxemia is baffling to physicians." American Journal of Respiratory and Critical Care Medicine 202.3 (2020): 356-360.

Xie, Jiang, et al. "Association between hypoxemia and mortality in patients with COVID-19." Mayo Clinic Proceedings. Elsevier, 2020.

Xia, Ye, and Gwendolyn Lee. "How to return to normalcy: Fast and comprehensive contact tracing of COVID-19 through proximity sensing using mobile devices.” arXiv preprint arXiv:2004.12576 (2020) 